\title{
Molecular Electronics - Integration of Single Molecules in Electronic Circuits
}

\author{
Marcel Mayor* and Heiko B. Weber*
}

\begin{abstract}
Electronic devices made with organic molecules may one day play an important role in integrated circuits. However, the research is still in its infancy. The mechanically controlled break-junction technique allows the measurement of the current through a single molecule junction. The current-voltage relations indicate a nonlinear conductance. Information about the junction can be provided by comparative studies of different molecules. It turns out that the conduction properties depend strongly on the molecular structure. This, in turn, encourages the idea of tailoring the electronic properties by an appropriate design of the molecule.
\end{abstract}

Keywords: Break-junction · Electron transport · Molecular electronics · Molecular wires .

Single-molecule studies

\section{Introduction and Brief Review}

For almost four decades, silicon-based integrated circuits have continuously reduced the feature sizes of devices. This miniaturization, described by Moore's law, has been mainly driven by the desire to reduce the cost per functional unit. However, in the last few years it became obvious that further decrease in feature size will become increasingly difficult due to physical limitations, accompanied by an enormous increase in expenses. This explains the growing interest for alternative concepts to build electronic devices.

A very promising approach is the integration of molecular structures to supplement specific functionalities on a semiconductor chip. The physical properties of such molecule-on-chip devices will depend strongly on the molecular structure. Consequently, the electronic function of the device may be given by careful design and tailor-made chemical synthesis. The huge

${ }^{*}$ Correspondence: Dr. M. Mayor and Dr. H.B. Weber Forschungszentrum Karlsruhe $\mathrm{GmbH}$

Institute for Nanotechnology

P.O. Box 3640

D-76021 Karlsruhe

Tel.: + 497247826392

Fax: + 497247825685

E-Mail: marcel.mayor@int.fzk.de;

heiko.weber@int.fzk.de

www.fzk.de/int/ potential of this approach is not only the very small size of molecules, giving rise to the hope for further reductions of the feature sizes on the chip. The production cost of a device with its electronic function defined by chemical synthesis may become much cheaper than a device with the corresponding function based on multi-step classical semiconductor production technology. However, currently this remains speculation as the technology to integrate and to address molecules or molecular assemblies on a chip has not yet been developed. But the research activity has strongly increased in the last few years [1], driven by some experimental breakthroughs.

The idea of profiting from molecular structures in order to perform electronic functions has its roots in the 1970s. In a pioneering theoretical paper Aviram and Ratner described the behavior of a molecule, consisting of two separated acceptor and donor $\pi$-systems, between two metallic electrodes [2]. Due to the energetic differences of the frontier orbitals of the two $\pi$-systems, they predicted the characteristics of a rectifier for such a device. Nearly two decades later, the proof of principle of this concept could be shown with donor-acceptor molecules organized in a Langmuir Blodget (LB) film [3]. Sandwiched between two metallic leads, the LBfilm displayed for a few cycles rectifying characteristics. Unfortunately, the rather weak van der Waals interaction of the molecules with the surface was insufficient to withstand the applied voltage, which inter- acts with the dipole moments of the molecules and destroys the ordered film. Recently, memory devices could be fabricated by deposition of tailor-made supramolecular systems like catenanes [4] or rotaxanes [5] as LB-films between electrodes. Electrochemically active components on the molecular building blocks of the supermolecule allow individual components to be addressed by voltage pulses. Thereby, changes in charge result in configurational changes of the supermolecule. The setup allows reversible switching between two discrete configurations of the supermolecule, which are shown in Scheme 1 for the catenane. As both configurations are stable at low voltage and have different tunneling characteristics, the actual state of the switch can therewith be read out at lower voltage.

For controlled use of molecular structures, a detailed understanding of the interplay between the structure and the electronic properties is crucial. Until the 1980s, such studies on molecular structures were limited to bulk properties [6]. With the development of scanning probe methods investigations of individual molecules became experimentally accessible [7]. For example, the conductance of individual conjugated molecular rods was qualitatively investigated by comparison of $\mathrm{AC}$ and DC STM studies on a gold surface covered with an insulating thioalkane self-assembled monolayer (SAM) and longer molecular rods, situated in defect positions of the SAM [8]. The common topological and conductance maxima were interpreted as 

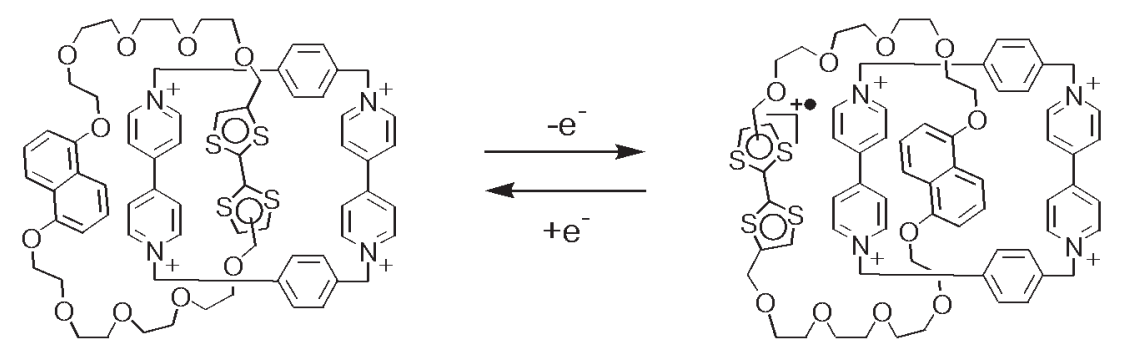

Scheme 1. A catenane molecule with two metastable conformations which can be converted by reversible reduction/oxidation, used in [4] for resistive memory devices. The device is formed by a Langmuir-Blodget film of these molecules, sandwiched between electrodes. The reduction/oxidation can then be steered by applying an appropriate voltage pulse $( \pm 2 \mathrm{~V})$. Thus information can be written on the device. As both conformations have different conductivities, the information can be read out at low voltages. the effects of the protruding conjugated rods. In another approach, the lateral extent of a SAM was limited by the use of pores in a silicon nitride membrane with a diameter of about $40 \mathrm{~nm}$. A SAM of 2'-amino-4ethynylphenyl-4'-ethynylphenyl-5'-nitro-1benzenethiol was deposited on a gold surface in a pore and covered with a gold electrode [9]. The device displayed a negative differential resistance (NDR: increase in voltage causes a decrease in current above a certain threshold) at temperatures below $60 \mathrm{~K}$. The device architecture and its curmatically represented in Fig. 1. This I/V characteristic turned out to be stable and reproducible. However, whether this unconventional behavior arises from the molecular structure or from the device architecture has not yet been clarified and is the topic of current investigations. with two electrodes have already been made. A first mechanically controlled break-junction (MCB) experiment was made with a fixed thin gold wire with a predetermined breaking point on a flexible substrate [10]. The gold wire was exposed to a para-dithiobenzene solution such that a SAM of these molecules was formed on the surface. By careful bending of the substrate while observing the current through the gold wire, the breaking of the wire could be determined. When the bending tension was released again, the two broken ends approach each other. At a given point, the first molecule is expected to touch the opposite side, forming thiol-gold bonds to both electrodes. The electric transport through this setup was investigated and interpreted as the transport through bridging molecules. To improve this MCB technique the gold wire was replaced by lithographically fabricated gold structures [11]. Terthiophenes, which were thiol functionalized on both ends, were immobilized between the two rent/voltage (I/V) characteristics are sche-

Attempts to contact a single molecule gold contacts and the I/V characteristics were investigated as a function of the electrode distance. Although these are both pioneering experiments, they lack any information about the number of molecules between both electrodes.

Our own experiments, which we present here, try to address several new topics. A further development of the MCB technique as tweezers for molecular structures is presented. Molecular rods are designed and synthesized in order to analyze the situation on a molecular level between the two electrodes of the MCB. To investigate the question whether the above-mentioned NDR originates from the molecular structure or from the device architecture, the corresponding molecule with two thiol functions was synthesized and investigated in a MCB. Additional information about the number of investigated molecules in a MCB was gained by synthesizing and investigating molecular rods with different symmetries along the rod axes. First correlation studies between the overall resistance of the electrode-molecule-electrode setup and the investigated molecular structure are presented. Theoretical studies accompany the experiments and support the design of future molecular structures with defined electronic functions.

\section{Mechanically Controlled Break- Junction (MCB)}

A single-molecule contact requires a pair of electrodes separated from each other by exactly the length of the molecule. The MCB technique provides a pair of electrodes with adjustable distance. The method is based on a lithographically fabricated gold structure consisting of two large areas connected with a thin gold bridge (thickness 20-50 nm). This gold structure is patterned on an insulating polyimide film on a flexible substrate. Reactive ion etching removes the polyimide around the gold structure, underetches the gold bridge and leaves it freely suspended. The final structure on the substrate is shown in Fig. 2 (left). The substrate is fixed in a three point setup that allows the substrate to be mechanically bent (Fig. 2, right). In a vacuum chamber the substrate is carefully bent while the electric resistance between the two large gold areas is monitored. Thereby, the thin gold bridge is elongated until it finally breaks, which is observed as an immediate increase of the resistance. Release of the bending tension allows the two broken ends of the gold structure to approach. The extremely flat architecture results in a distance resolution of the two electrodes of

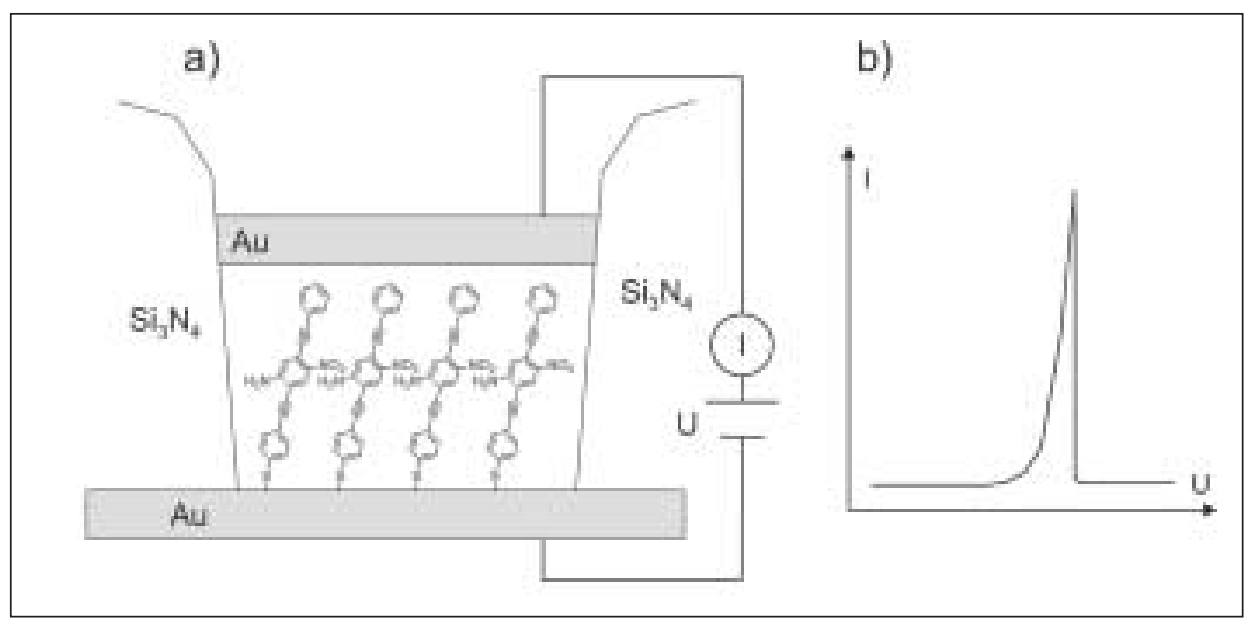

Fig. 1. a) Sketch of the experiment in which a self-assembled molecular film is sandwiched between two gold films [9]. This film is placed in a nanopore in a $\mathrm{Si}_{3} \mathrm{~N}_{4}$ membrane, which restricts the lateral dimension of the device. b) These devices show an unusual I/V relation at low temperatures: the current is strongly suppressed at low voltages. At $V \sim 2 \mathrm{~V}$, the current suddenly increases strongly and breaks down. The phenomenon that the current decreases when the voltage is increased is called negative differential resistance (NDR). 
better than a tenth of an Angstrom. The setup is therefore ideal to immobilize molecular rods with lengths in the order of a few nanometers.

\section{Integration of Single Molecules in Electronic Circuits by MCBs}

So far, no microscopy technique is suitable to analyze the situation between the two electrodes of a MCB. Information can only be gained by analysis of the conductance data and comparison between appropriately designed different molecules. A basic requirement for the molecule design is a rod-like structure with two thiol endgroups for covalent linkage on the gold surfaces. The experiment discussed above, displaying a NDR for a SAM of a rod-like molecule, inspired our first investigations. As the molecular rod of the SAM was thiolfunctionalized on one side only, the connection of the SAM to the top electrode is not clear and may be the origin of the observed unexpected I/V behavior. To investigate the current transport through this structure in further details and to address the question whether the observed NDR ori-

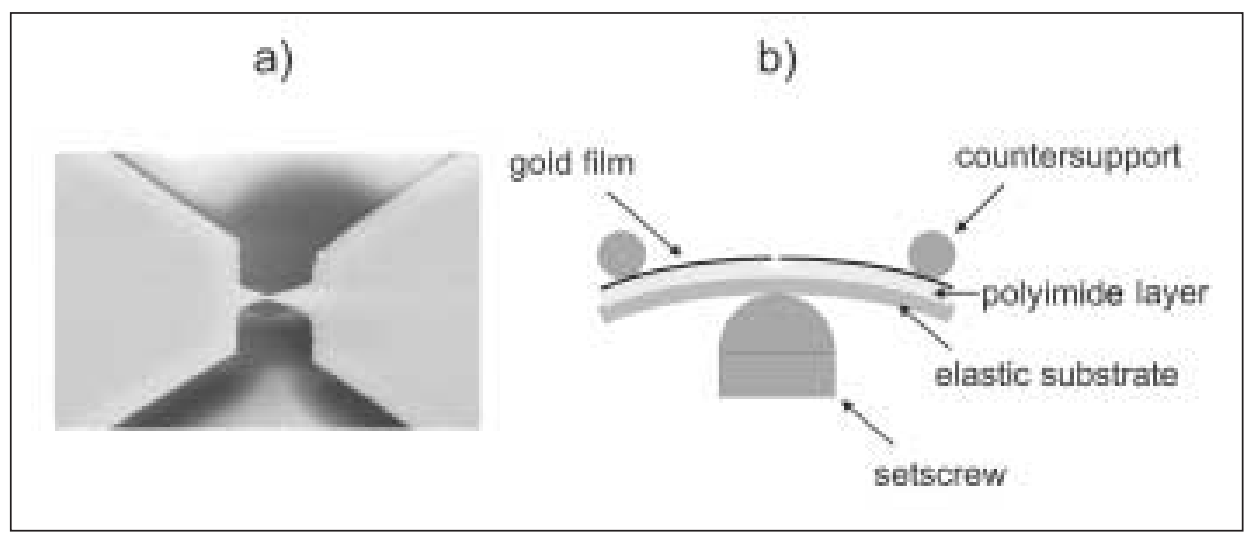

Fig. 2. The mechanically controlled break-junction setup. Left panel: SEM-picture of a lithographically produced gold film. The designated breaking point (in the center of the picture, 50 $\mathrm{nm}$ large) is a freely suspended gold bridge which forms the electrode pair once it is broken. Right panel: Sketch of the bending mechanism. The setscrew is moved upwards to bend the flexible substrate until the gold bridge on top breaks. By bending back and forth, the electrode gap can be tuned with sub-Ångstrom resolution.

gins in the molecules' structure or the device architecture, the corresponding rodlike structure 1 with acetyl-protected thiol functions on both ends was synthesized (Scheme 2). 1 is a stiff and conjugated $\pi$-system, consisting of three benzene cores connected with acetylene bridges. The two acetyl-protected thiol functions are separated by about $2 \mathrm{~nm}$. The acetyl-protected amino function and the nitro function in the central benzene ring of $\mathbf{1}$ result in a dipole moment along the rod axis. For a single molecule immobilized between two electrodes, a dipole moment along the rod axis

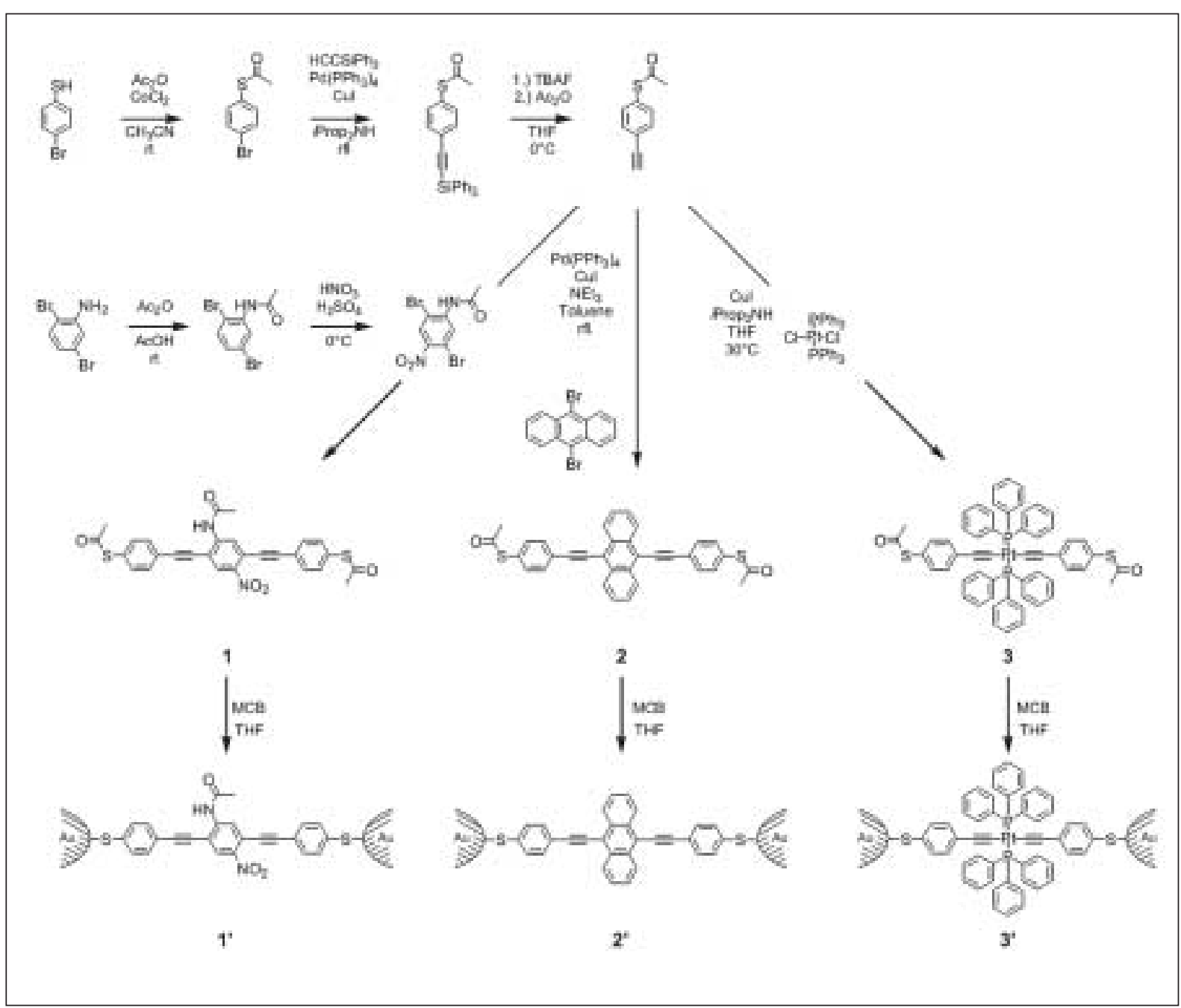

Scheme 2. Synthesis of the molecular rods 1,2 and 3. 
may result in a dependence of the I/V characteristics on the direction of the applied current.

Of particular interest is a control experiment with an immobilized molecular rod having a symmetry plane perpendicular to the molecules axis through its center. For such a setup similar I/V characteristics in both current directions would be expected. The molecular rod 2 was synthesized for this purpose. With two acetyl-protected thiol functions on both ends, 2 has an inversion center in its center. However, in its immobilized form, $\mathbf{2}$ ' has a symmetry plane perpendicular to its rod-axis. Both molecules were synthesized with acetyl-protected thiol functions. This protection group is known to deprotect spontaneously on gold surfaces and to slow down the kinetics of SAM formation [12]. Both molecular rods were investigated repeatedly in a MCB [13].

The broken MCB was opened to about $10 \mathrm{~nm}$ and a $5 \times 10^{-4} \mathrm{M}$ solution of the molecules in THF was applied for $30 \mathrm{~s}$. After extensively rinsing with THF under $\mathrm{N}_{2}$ atmosphere, the sample chamber was evacuated to $\sim 5 \times 10^{-7}$ mbar. At an applied voltage of about $1 \mathrm{~V}$ the two electrodes were slowly brought closer together. At this stage of the experiment we assume that individual molecules are immobilized on the gold surface of one electrode with the thiol function on one end, while the thiol function on the opposite side is still acetyl protected. The rod-like structures consisting of conjugated $\pi$-systems orient themselves in the strong electric field towards the electrode on the opposite side. When the electrode gap is closed further, a sudden rise in the current is observed. This current is insensitive to small changes in distance, displaying a fairly stable lock-in situation, probably due to the first molecule bridging the two electrodes. All displayed I/V curves are recorded in this locked-in situation. Further approach of the electrodes results in a strong increase of the current. Sometimes, a second plateau with doubled current could be observed and was interpreted as the contribution of a second molecule bridging the gap.

The I/V curves of $\mathbf{1}$ ' and $\mathbf{2}$ ' observed at room temperature in the lock-in state are displayed in Fig. 3. Comparable overall resistances through the setup of the order of $1 \mathrm{M} \Omega$ at $1 \mathrm{~V}$ were observed for both molecules. This was expected, as both rod-like structures consist of comparable conjugated $\pi$-systems, namely in para-position ethynyl-connected aromatic systems. While the I/V characteristics of the symmetric rod 2 ' was not affected by current inversion, 1' displayed substantial differences upon current inversion (Fig. 3). With the rod symmetry an intrinsic property of the molecular structure, for the first time a molecular property was reflected and identified in the I/V characteristics. In addition, symmetric I/V curves were recorded in the locked-in state only for the symmetric molecular rod 2', while only asymmetric I/Vs were observed with the asymmetric rod 1'. This indicates, among other arguments, that most likely a single molecular rod is bridging the two electrodes in the lock-in situation. If the junction was formed by many molecules in parallel with random orientation, one would rather expect a symmetric I/V characteristic.

A sudden breakdown of the current, as described for the SAM device (Fig. 1; [9]) at temperatures below $60 \mathrm{~K}$ with a comparable molecular structure, was not observed for the asymmetric rod 1'. As our experiments were recorded at room temperature, this may be a possible explanation for the lack of NDR. However, very recent experiments at temperatures below $30 \mathrm{~K}$ have also failed to display an NDR. This suggests that the described NDR results from the architecture of the device and does not have its origin in the molecular structure alone.

Quantum chemical studies of the investigated systems reproduce the phenomenology observed in the experiments. To take the influence of the electrodes approximatively into account, a $\mathrm{Au}_{29}$ cluster was attached on both sides of the molecule (Fig. 4). This supermolecule is still tractable in DFT calculations. The main interest was dedicated to charge redistribu-
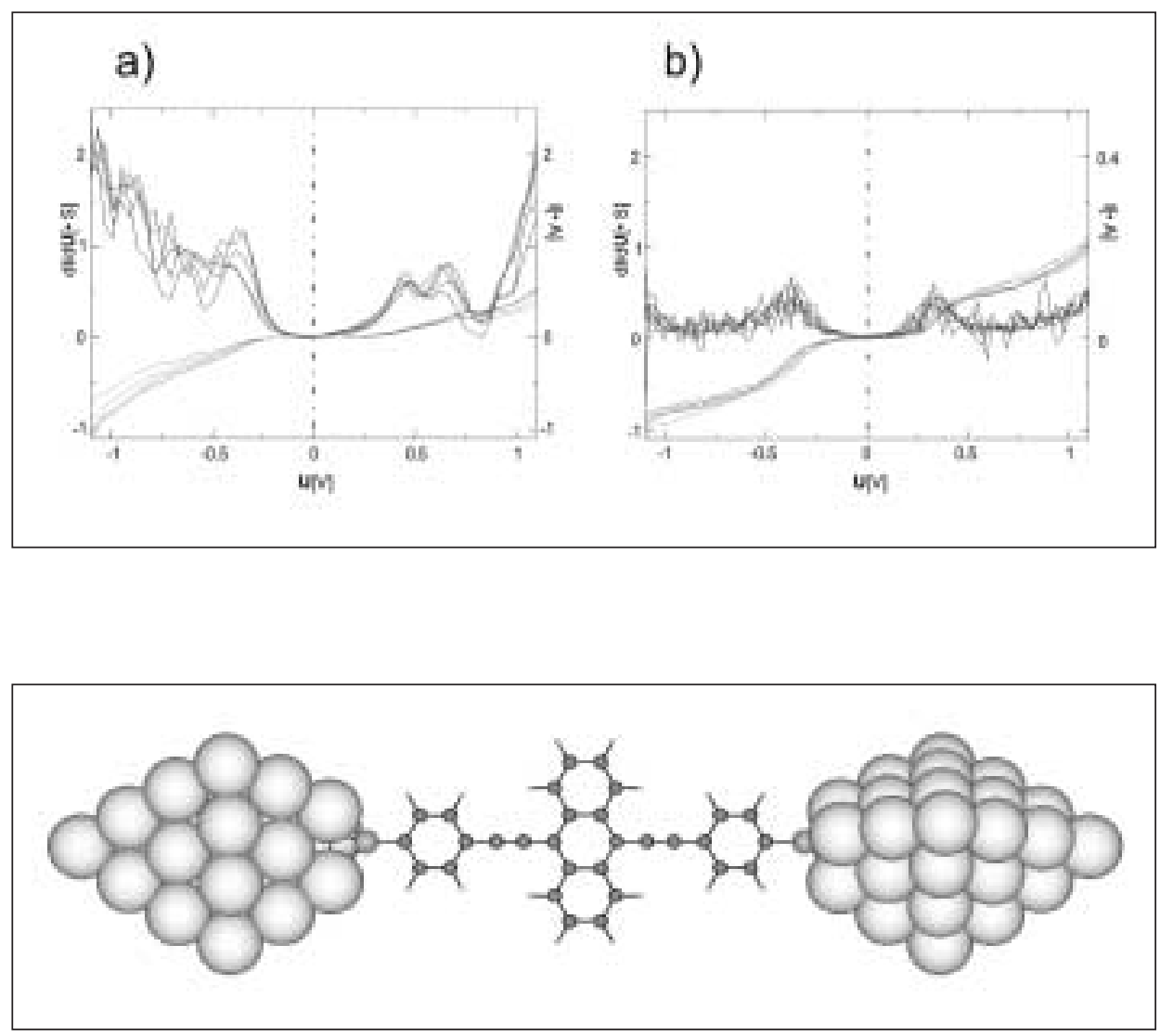

Fig. 3. a) Current-voltage relations (I/V: grey lines) and differential conductance (dl/dU: black lines) of the molecular junction 1'. The conductance is clearly nonlinear and asymmetric with respect to current inversion (positive voltages correspond to one current direction through the molecule, negative voltages to the opposite). The peak-like structures in $\mathrm{dl} / \mathrm{dU}$ probably indicate transport through molecular orbitals. b) The corresponding measurement with $2^{\prime}$. The data are (in good approximation) symmetric with respect to current inversion. The comparison of both measurements proves that the I/Vs reflect intrinsic properties of the molecules: their symmetry.

Fig. 4. To compute the properties of the single-molecule junction, a DFT calculation was performed with the symmetric molecule $\mathbf{2}^{\prime}$ attached to two $\mathrm{Au}_{29}$ clusters, which simulate the influence of the electrodes. 
tion in the presence of finite electric fields, which correspond to the applied voltage. It turns out that the polarizability of the supermolecule, which is related to the conductivity, behaves in a qualitatively similar fashion to the experimental observations [14].

\section{Correlation of Molecular Structure vs. Electronic Properties}

The above experiment comparing the I/V characteristics with the molecule symmetry already demonstrated the dependence of electronic properties on the molecular structure. However, even though the differences in symmetry were reflected by the observed I/Vs, the overall electronic properties like threshold voltage of the conductivity and resistance of the device with the integrated molecule were comparable. Electronic transport is expected through one of the frontier orbitals of the $\pi$-system. As both rods $\mathbf{1}$ and $\mathbf{2}$ are composed of similar components (ethynyl-connected aromatic systems) with similar connections (para-position for acetylenes and thiols), their conjugated $\pi$-systems are comparable and similar electronic properties are not at all surprising.

But what happens if the conjugation on a molecular rod is interrupted? If the electronic transport is through a frontier orbital of the $\pi$-system, such a molecular rod consisting of two divided $\pi$-systems should display different electronic properties. Naïvely, one would expect that the I/V characteristics are dominated by the interrupting structure, which is the bottleneck for electronic transport. Platinum(II) ions are well-known rigid linkers in acetylene scaffolding [15]. Spectroscopic investigations of trans-platinum(II)-linked acetylene structures have shown the pure $\sigma$-character of the acetylene-carbon-platinum(II) bond and hence the potential of the platinum(II) ion as conjugation passive linker or interrupter of the $\pi$-system. Therefore, the metalorganic trans-platinum(II) ethynyl complex 3 was designed and synthesized by the conversion of trans-bis(triphenylphosphine)platinum(II) chloride with (4-ethynyl)phenyl thioacetat [16], as shown in Scheme 2. The resulting molecular rod $\mathbf{3}$ has again acetyl-protected thiol functions at both ends that allow immobilization between two gold electrodes. 3 crystallizes from chloroform in single crystals, suitable for X-ray analysis (Fig. 5). The platinum ion is coordinated in a square-planar arrangement by two trans-triphenylphosphines and trans-acetylene ligands, resulting in a sulfur-to-sulfur distance of $1.83 \mathrm{~nm}$.

To investigate the electronic properties of $\mathbf{3}$, similar protocols as for $\mathbf{1}$ and $\mathbf{2}$ were used. It turned out that after deposition of $\mathbf{3}$ on the electrodes, the applied voltage while approaching the two electrodes had to be set at a higher value of $\sim 4 \mathrm{~V}$. Again a stable lock-in situation was observed at room temperature and reproducible I/V curves were recorded. Further approach of the electrodes occasionally resulted in a doubling of the current, probably due to a second molecule in the break-junction. The strong analogy to the behavior of $\mathbf{1}$ and $\mathbf{2}$ in the break-junction leads to the assumption that most likely a single molecular rod $\mathbf{3}$ is bridging the two electrodes in the locked-in state. The I/V curves recorded for $\mathbf{3}^{\prime}$ are shown in Fig. 6. They are symmetric with respect to voltage inversion and display an exponential increase of the current with ap- plied voltage. The overall resistance of the electrode-3'-electrode device is with 5-50 $\mathrm{G} \Omega$ about three orders of magnitude larger than observed for the conjugated rods $\mathbf{1}^{\prime}$ and 2 ', pointing out the potential of the trans-platinum(II) connection as mechanically rigid but electronically interrupting linker in molecular systems. The I/V relationship of $\mathbf{3}^{\prime}$ resembles the behavior of a classical insulator and can be described by a quantum-mechanical model for a rectangular tunnel barrier with a tunnel barrier height of $2.5 \mathrm{eV}$ (circles in Fig. 6).

\section{Summary, Conclusion and Perspective}

The results point out that individual molecules can be contacted with the MCB technique. Together with tailor-made chemical synthesis, the combination becomes a beautiful tool to investigate the correlation between molecular structure and electronic property in detail. The substantial effect of the molecular structure on the electronic properties that has already been demonstrated indicate the potential of molecules in future hybrid devices as functional units. Currently, we are focusing on new molecular structures, designed and synthesized to perform specific electronic functions. Among them are molecular rectifiers, molecular memory devices and molecular rods that may display NDR effects. The studies presented here are still on a basic research level and contribute to the understanding of a detailed structure-property correlation. The methods may even allow the study of critical parameters of integrated molecules like stability or fatigue properties. However, to profit from the accumu-

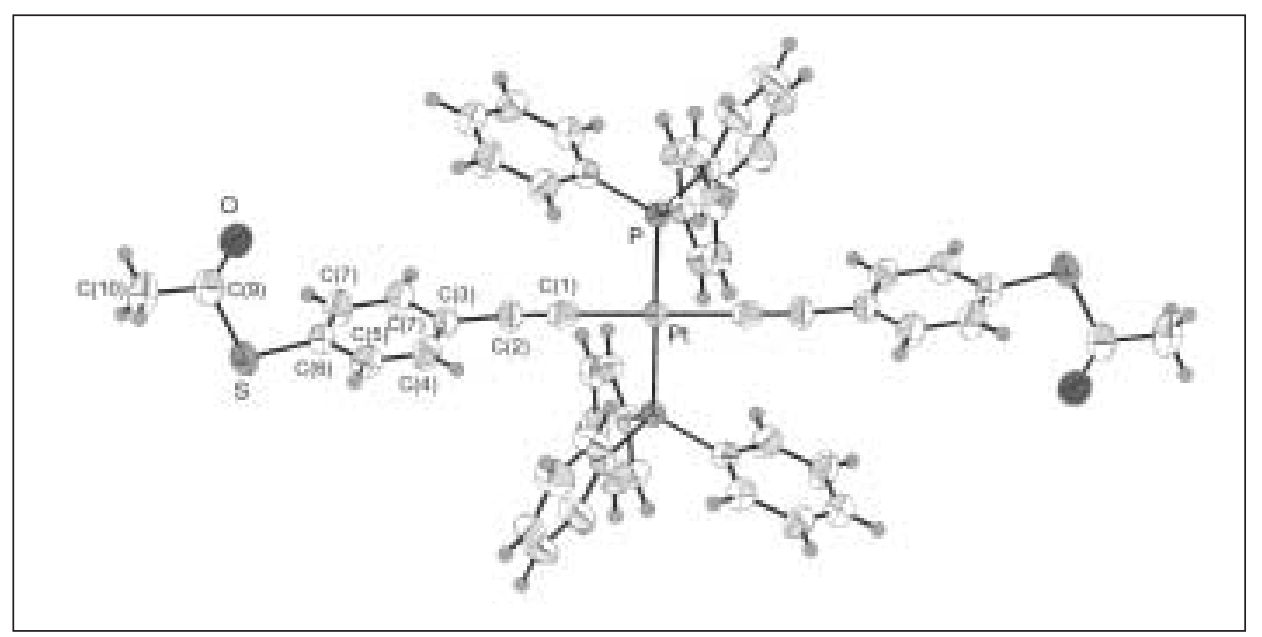

Fig. 5. Molecular structure of $\mathbf{3}$ in the crystal, obtained from $\mathrm{X}$-ray structure analysis. 


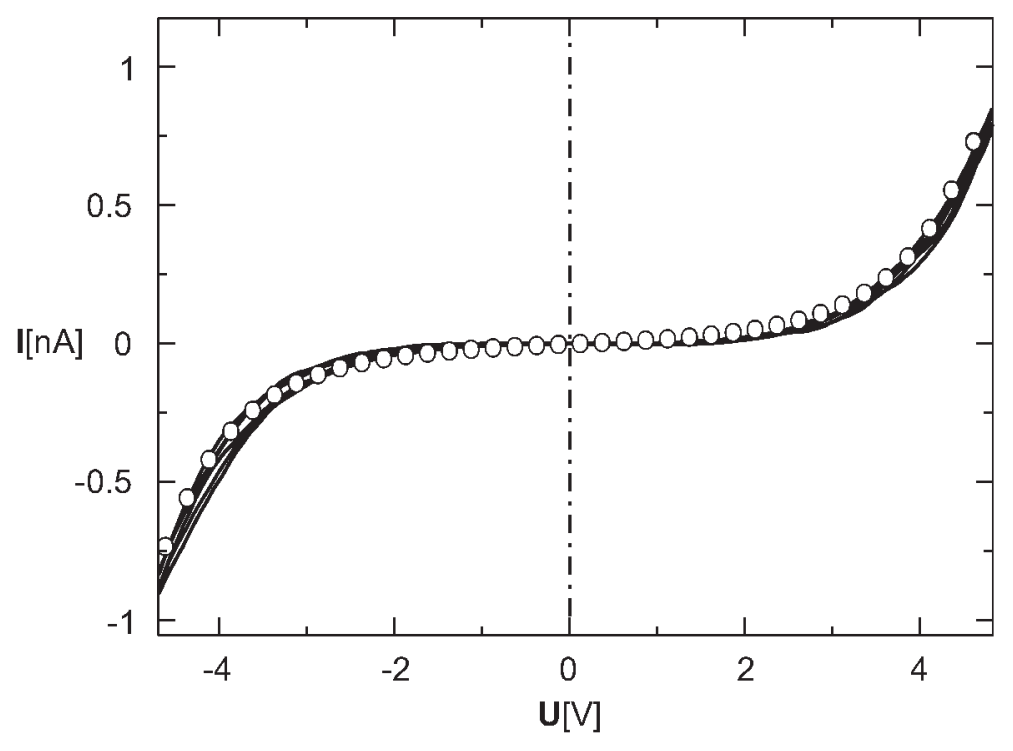

Fig. 6. The current-voltage relation of $\mathbf{3}^{\prime}$ (lines). The resistance of the device with $\mathbf{3}^{\prime}$ is more than three orders of magnitude higher than with $\mathbf{1}^{\prime}$ and $\mathbf{2}^{\prime}$. The shape of the I/V indicates insulating behavior. The circle symbols are theoretical data, which assume a simple rectangular potential barrier with a barrier height of $2.5 \mathrm{eV}$.

lated knowledge and to integrate molecular structures in semiconductor technologies is still a huge challenge. The parallel integration and addressing of larger numbers of molecules is with the methods presented here not achievable [17] and future innovative ideas and concepts are sought to overcome this drawback.

\section{Acknowledgement}

We gratefully acknowledge D. Beckmann, J. Reichert, R. Ochs, H. v. Löhneysen, C. v. Hänisch, F. Weigend, R. Ahlrichs, M. Fischer, M. Di Leo, C. Didschies, M. Elbing, J.M. Lehn, S.T. Balaban, M. Hettler, O. Wollersheim and the Strategiefond from the Helmholtz Foundation for financial support.

Received: August 28, 2002

[1] For an overview: C. Joachim, J.K. Gimzewski, A. Aviram, Nature 2000, 408, 541-548; M.A. Reed, J.M. Tour, Scientific American 2000, 282, 86-93; 'Molecular Electronics: Science and Technology', Ed. A. Aviram, M. Ratner, Ann. N. Y. Acad. Sci. New York, 1998, 852; 'Molecular Electronics II' Ed. A. Aviram, M. Ratner, V. Mujica, Ann. N. Y. Acad. Sci. New York, 2002, 960 .

[2] A. Aviram, M.R. Ratner, Chem. Phys. Lett. 1974, 29, 277-283.

[3] G.J. Ashwell, Thin Solid Films 1990, 186, 155-165.

[4] C.P. Collier, G. Mattersteig, E.W. Wong, Y. Luo, K. Beverly, J. Sampaio, F.M. Raymo, J.F. Stoddart, J.R. Heath, Science 2000, 289, 1172-1175.
[5] Y. Luo, C.P. Collier, J.O. Jeppesen, K.A. Nielsen, E. Delonno, G. Ho, J. Perkins, H.-R. Tseng, T. Yamamoto, J.F. Stoddart, J.R. Heath, ChemPhysChem 2002, 3, 519-525.

[6] For an overview: 'Electronic Materials: The Oligomer Approach', Ed. K. Müllen, G. Wegner, Wiley-VCH, Weinheim, 1998.

[7] G. Binning, H. Rohrer, C. Gerber, E. Weibel, Phys. Rev. Lett. 1982, 49, 57-61.

[8] L.A. Bumm, J.J. Arnold, M.T. Cygan, T.D. Dunbar, T.P. Burgin, L. Jones II, D.L. Allara, J.M. Tour, P.S. Weiss, Science 1996, 271, 1705-1707.

[9] J. Chen, M.A. Reed, A.M. Rawlett, J.M. Tour, Science 1999, 286, 1550-1552.

[10] M.A. Reed, C. Zhou, C.J. Mullen, T.P. Burgin, J.M. Tour, Science 1997, 278, 252-254.

[11] C. Kegueris, J.-P. Bourgoin, S. Palacin, D. Esteve, C. Urbina, M. Magoga, C. Joachim, Phys. Rev. B 1999, 59 , 12505-12513.

[12] J.M. Tour, L.R. Jones II, D.L. Pearson, J.J.S. Lamba, T.P. Burgin, G.M. Whitesides, D.L. Allara, A.N. Parikh, S.V. Atre, J. Am. Chem. Soc. 1995, 117, 9529-9534.

[13] J. Reichert, R. Ochs, D. Beckmann, H.B. Weber, M. Mayor, H. v. Löhneysen, Phys. Rev. Lett. 2002, 88, 176804-1-176804-4.

[14] H.B. Weber, J. Reichert, F. Weigend, R. Ochs, D. Beckmann, M. Mayor, R. Ahlrichs, H. v. Löhneysen, Chem. Phys. 2002, 281, 113-125.

[15] P. Siemsen, U. Gubler, C. Bosshard, P. Günter, F. Diederich, Chem. Eur. J. 2001, 7, 1333-1341.

[16] M. Mayor, C. v. Hänisch, H.B. Weber, J. Reichert, D. Beckmann, Angew. Chem. Int. Ed. 2002, 41, 1183-1186.

[17] The integration of LB films between crossbar electrodes as discussed in [4] and [5] are first steps towards parallel integration. 